\title{
Inhibited Spontaneous Emission in Solid-State Physics and Electronics
}

\author{
Eli Yablonovitch \\ Bell Communications Research, Navesink Research Center, Red Bank, New Jersey 07701
}

(Received 23 December 1986)

\begin{abstract}
It has been recognized for some time that the spontaneous emission by atoms is not necessarily a fixed and immutable property of the coupling between matter and space, but that it can be controlled by modification of the properties of the radiation field. This is equally true in the solid state, where spontaneous emission plays a fundamental role in limiting the performance of semiconductor lasers, heterojunction bipolar transistors, and solar cells. If a three-dimensionally periodic dielectric structure has an electromagnetic band gap which overlaps the electronic band edge, then spontaneous emission can be rigorously forbidden.
\end{abstract}

PACS numbers: $42.50 .-\mathrm{p}, 42.55 . \mathrm{Bi}, 78.45 .+\mathrm{h}$

It has been recognized for some time ${ }^{1}$ that the spontaneous emission by atoms is not necessarily a fixed and immutable property of the coupling between matter and space, but that it can be controlled by modification of the properties of the radiation field. Recently there have been experiments in which the spontaneous emission by Rydberg atoms ${ }^{2}$ and by Penning-trapped electrons ${ }^{3}$ has been inhibited in a microwave cavity which had no electromagnetic modes at the transition frequency. Such a capability is no less important in the solid state where spontaneous emission in the form of electron-hole radiative recombination plays a decisive role. For direct-gap semiconductors it is commonplace ${ }^{4}$ for the internal spontaneous emission quantum efficiency of electron-hole recombination to exceed $90 \%$ even at room temperature.

Therefore I suggest that the direct-gap semiconductors are a natural physical system for the study of inhibited spontaneous emission. There is ample motivation since the performance of semiconductor lasers, heterojunction bipolar transistors, and solar cells are all limited by spontaneous emission, but each in a characteristically different way. In this paper, I analyze the different aspects of inhibited spontaneous emission appropriate to each of these cases.

A starting point for this discussion is the observation by Einstein that spontaneous emission must inescapably coexist with absorption and stimulated emission. It will be neither feasible nor desirable to eliminate spontaneous emission entirely if the function of the semiconductor happens to be the emission or the absorption of light itself. Examples of this would be the semiconductor laser and the solar cell, respectively. In those cases the goal would be to restrict the spontaneous emission to only those electromagnetic modes which are absolutely necessary. In other functions such as the heterojunction bipolar transistor, where the radiation field would preferably be absent, all spontaneous emission should be suppressed if possible.

Periodic structures have been playing an increasingly important role in semiconductor lasers. For example, in distributed-feedback lasers ${ }^{5}$ the index of refraction is periodically modulated along the laser axis. In wave optics, layered structures (interference coatings) in which the index of refraction alternates from high to low every quarter wavelength have also become indispensable. The periodic spatial modulation opens up a forbidden gap in the electromagnetic dispersion relation, at least for light propagating perpendicular to the layers. The prohibition of wave propagation in the forbidden gap makes these periodic dielectric structures valuable as highly ideal reflective mirrors, for example, for use in Fabry-Perot resonators. We can anticipate, then, that full threedimensional spatial periodicity of $\lambda / 2$ in the refractive index can result in a forbidden gap in the electromagnetic spectrum near the wavelerigth $\lambda$ irrespective of propagation direction, just as the electronic spectrum has a band gap in crystals. If the electromagnetic band gap overlaps the electronic band edge by at least a few $k T$ in energy, then electron-hole radiative recombination will be severely inhibited. Such an inhibition can be much more complete than in a metallic waveguide ${ }^{2}$ since dielectric structures can in principle be lossless. Therefore it would be very interesting to create periodic three-dimensional dielectric structures in which there exists an electromagnetic band gap.

The recombination rate of electrons and holes in a semiconductor can be expressed as a power series in the injected carrier density $n$,

$$
d n / d t=-\left(n / \tau+B n^{2}+C n^{3}\right),
$$

where $\tau$ is the nonradiative lifetime due to recombination at defects in the bulk or on the surface, Bnp is the twobody radiative recombination rate which we seek to modify, and $C$ is the combined electron and hole threebody Auger recombination coefficient which is intrinsic to the material. In Eq. (1) it is assumed that the doping density is negligible compared to the injected density so that $n=p$. From Eq. (1) the radiative quantum efficiency has a maximum value $\left[1+(2 / B)(C / \tau)^{1 / 2}\right]^{-1}$ 
at an injection density $n=1 /(\tau C)^{1 / 2}$. In today's highquality ${ }^{6} \mathrm{AlGaAs} / \mathrm{GaAs}$ double heterostructure epilayers the defect density is low enough that $\tau \sim 2 \mu$ sec. The ordinary spontaneous-emission coefficient is $B=10^{-10}$ $\mathrm{cm}^{-3} \mathrm{sec}^{-1}$, and the Auger coefficient is $C \sim 10^{-29}$ $\mathrm{cm}^{-6} \mathrm{sec}^{-1}$. For these parameters, at an optimal injection level around $n=2 \times 10^{17} \mathrm{~cm}^{-3}$ the internal quantum efficiency is $\approx 0.96$ showing the importance of spontaneous emission as the dominant recombination channel even at room temperature. This recombination radiation is emitted in a narrow spectral range within thermal energy $k T$ of the electronic band edge.

At the lasing threshold, spontaneous emission represents the major parasitic current sink. If the electronic band edge happens to fall within an electromagnetic band gap, not only would spontaneous emission be suppressed but also stimulated emission would be absent. In distributed-feedback lasers ${ }^{5}$ this problem is already evident. There is periodic modulation along the laser axis and therefore a forbidden gap in the electromagnetic dispersion relation. Nevertheless the laser is meant to lase in the center of the forbidden gap!

To understand how this comes about it is best to consider the modes of a Fabry-Perot resonator, which is constructed in the usual manner of quarter-wave multilayer dielectric mirrors. In such a resonator the mirrors face each other and are separated by an integral number of half wavelengths. Figure 1 shows such a Fabry-Perot resonator in which the mirrors happen to be one-half wavelength apart. The net effect of the left and right mirrors facing each other is that they combine to form an overall periodic dielectric structure, but with a quarter wavelength of phase slip introduced into the very center. This same quarter wavelength of phase slip is often employed in distributed-feedback lasers, effectively converting them into Fabry-Perot resonators. The standing-wave mode is sometimes ${ }^{7}$ regarded as a bound state split off into the electromagnetic band gap and localized to the quarter-wave defect in the periodic structure. Our goal is to create and shape a local electromag-

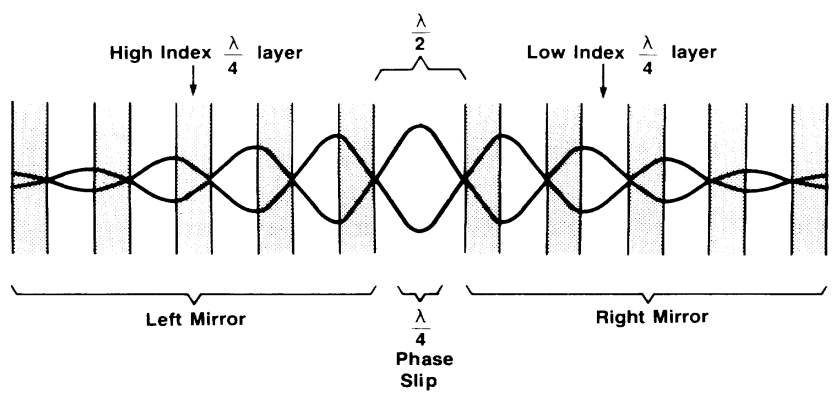

FIG. 1. A Fabry-Perot resonator made of multilayer dielectric mirrors with a space of one-half wavelength between the left and right mirrors. The net effect is to introduce $\lambda / 4$ phase slip in the overall periodic structure. netic mode in the forbidden gap by introducing phase slip, not merely along the laser axis but also in the two orthogonal directions to provide lateral confinement as well. This local electromagnetic mode which is shaped to follow the thin active gain layer will become the lasing mode. This will fulfill our goal to severely curtail the overall spontaneous emission into the electromagnetic band gap while nevertheless permitting it to proceed into a specific lasing mode as it inevitably must.

Following Purcell, ${ }^{1}$ we can now estimate the degree of reduction of spontaneous emission. The density of electromagnetic modes per unit frequency per unit volume in the normal crystal is $8 \pi n^{2} v^{2} / c^{3}$. For the case of a local electromagnetic mode, there will be but one mode in the spontaneous emission bandwidth $\Delta v_{\mathrm{sp}}$, in the mode volume $V$. The reduction factor of spontaneous emission is

$$
\lambda^{2} c / 8 \pi \Delta v_{\mathrm{sp}} V
$$

By appropriate of introduction phase slips into the periodic electromagnetic structure, the mode volume can be made sufficiently larger than a cubic wavelength and the reduction of spontaneous emission will then be substantial. When this is combined with modern approaches ${ }^{8}$ for reducing nonradiative recombination, the laser threshold current density may well drop below $1 \mathrm{~A} / \mathrm{cm}^{2}$.

A specific realization of the desired structure is illustrated in Fig. 2(a). Instead of one-dimensional corrugations as in distributed-feedback lasers, deep corrugations are etched along two surface directions leaving in effect a checkerboard pattern on the substrate. The height, width, and depth of each cube-shaped etch pit is $\lambda / 2\left(n_{1}+n_{2}\right)$ or, in other words, one-quarter the average material wavelength where $\lambda$ is the vacuum wavelength, and $n_{1}$ and $n_{2}$ are the two indices of refraction. As shown in Fig. 2(b), further epitaxial layers are each grown one-quarter wavelength thick under such conditions as to minimize the step coverage and maintain the cubic structure with each successive layer. The result of such a three-dimensional crystal growth is a facecentered-cubic (fcc) structure in real space, but on a length scale which is about a thousand times larger than the fcc crystal structure of the atoms. The threedimensional periodicity of the index of refraction leads to an fcc reciprocal lattice for electromagnetic waves with the classic Brillouin zone as illustrated in Fig. 2(c). With a sufficiently large difference in refractive index between $n_{1}$ and $n_{2}$, a gap will open up in the electromagnetic density of states. The idea here is for the gap or minimum in the density of electromagnetic states to overlap the maximum in the spontaneous emission spectrum.

In Fig. 2(b), the active gain region resides in an $n_{2}$ refractive index layer which is thicker than normal in order to provide some phase slip for vertical confinement of the active mode. The lasing mode need not reside in the very 


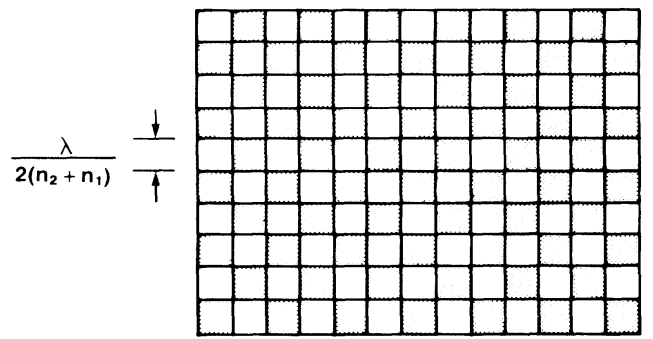

(a)

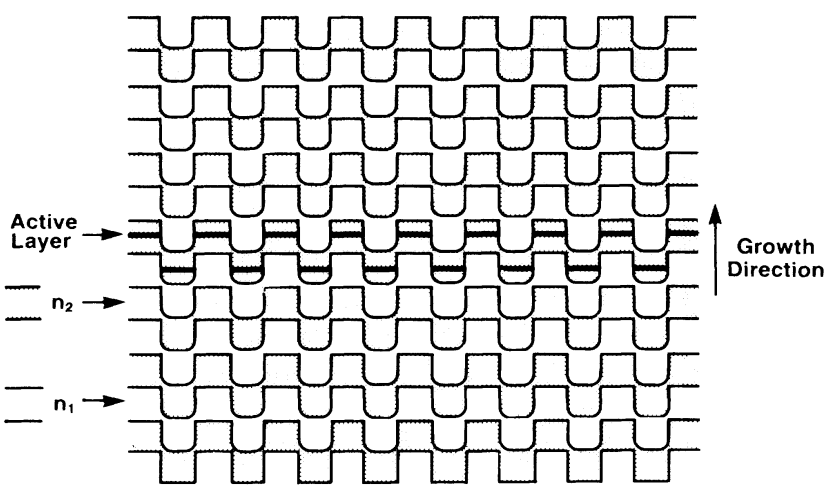

(b)

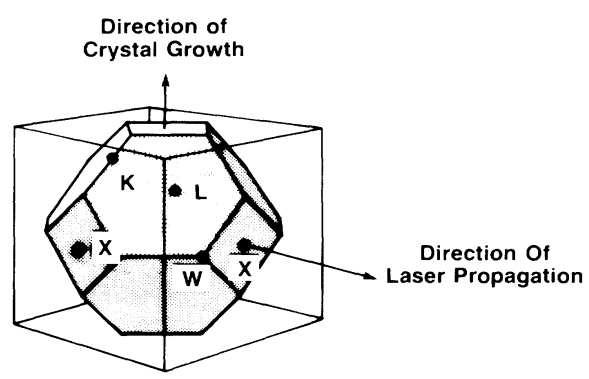

(c)

FIG. 2. (a) Top view of a substrate which has been patterned into a periodic checkerboard pattern with cubic etch pits one-quarter wavelength in length. (b) Side view of the grown structure with an active laser-gain layer and some phase slip to provide vertical optical confinement. In three dimensions, the overall periodic structure of the dielectric constant is face-centered cubic. (c) The fcc Brillouin zone in the electromagnetic reciprocal space. The laser propagation is shown in the $X$-point direction of reciprocal space, but the design can be changed to permit lasing into an arbitrary direction.

center of the forbidden electromagnetic gap. It may be tuned to one edge of the gap or indeed below the actual gap since laser gain first appears on the red side of the spontaneous emission spectrum. This tuning may be accomplished by a weak index modulation at a period different from the principal index modulation, and purely for the purpose of laser feedback rather than inhibition of spontaneous emission. This weak index modulation would control the length of the lasing mode permitting a good match to the available gain and permitting a sufficiently large mode volume.

A lower limit can now be placed on the minimum refractive index difference required to assure that a gap will open up in the electromagnetic spectrum. At the point $X$ in the Brillouin zone, the gap will be centered at a frequency $v=n_{\text {av }} c / \lambda$, while at the $L$ point the gap will be centered at a lower frequency $v=(\sqrt{3} / 2) n_{\mathrm{a} v} c / \lambda$. This is because the $L$ point is closer to the center of the zone than the $X$ point. It is important that the two gaps should overlap to assure a forbidden gap rather than merely a density of states minimum. In the onedimensional case ${ }^{9}$

$$
\Delta v_{\text {gap }}=(2 / \pi) v \Delta n / n
$$

The overlap of the gaps at the $X$ and $L$ points of the Brillouin zone requires that

$$
\Delta v_{\text {gap }}=(1-\sqrt{3} / 2) v
$$

Satisfying these two conditions implies a lower limit $\Delta n \approx 0.21 n$ where $\Delta n=n_{2}-n_{1}$. Similarly a stricter limit can be found by comparing the $W$ and $L$ points. In general these limits should be easy to satisfy, but it may be difficult to find a common lattice-matched pair of materials with a sufficiently large index difference. Nevertheless we may conclude that inhibited spontaneous emission is a real possibility in semiconductor lasers, but that it may require further materials development before the benefits are fully felt. In the meantime the concept of the electromagnetic band gap can be explored in such experimental systems as hexagonal-close-packed glass or polystyrene spheres.

We turn now toward the role of spontaneous emission in heterojunction bipolar transistors and solar cells. As before, the idea is to minimize electron-hole recombination especially in the base region of transistors. The current gain of the transistors would then be enhanced. As it happens, there is a conflicting requirement of low series resistance for high speed operation and the base is often so heavily doped that nonradiative Auger recombination becomes dominant. Therefore the application of inhibited spontaneous emission would be limited to only those cases of moderate base doping.

In solar cells radiative emission does indeed play the key role, but as with lasers it cannot be completely eliminated since it must coexist with light absorption from the surrounding space. Nevertheless there is significant potential reduction available, since the density of electromagnetic modes in the surrounding space is only $8 \pi v^{2} / c^{3}$, while the final-state density inside the semiconductor is $n^{2}$ times greater. While the external spontaneous emission is actually needed to balance the absorption, the $n^{2}$-times-larger internal spontaneous emission is completely unnecessary. Only the spontaneous emission within the escape cone for total internal reflection con- 


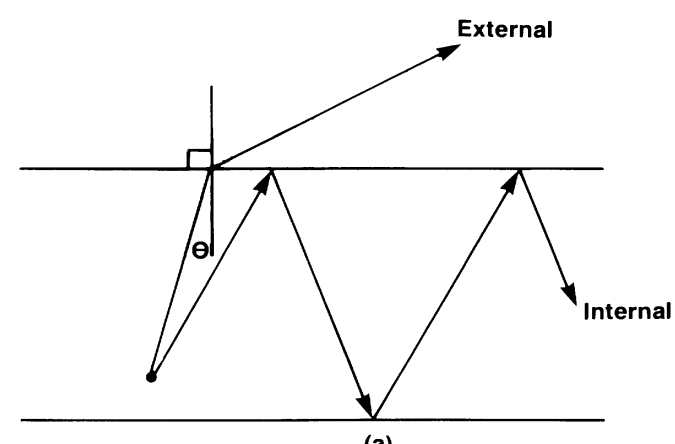

(a)

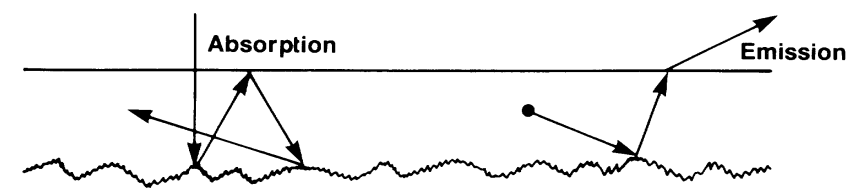

(b)

FIG. 3. (a) In a solar cell with plane parallel geometry the internal spontaneous emission can be trapped and eventually reabsorbed. (b) A much more practical geometry with a roughened rear surface. Externally incident radiation is absorbed in a much thinner layer as a result of light trapping. Roughness out-couples the internal spontaneous emission, but now the thinner semiconductor implies correspondingly less emission per unit area.

tributes externally, the balance being trapped internally:

$$
I_{\mathrm{ext}}=2 \int_{0}^{\theta_{c}} I_{\mathrm{int}} \cos \theta \frac{2 \pi \sin (\theta)}{4 \pi} d \theta=\frac{I_{\mathrm{int}}}{n^{2}}
$$

where $n \sin \left(\theta_{c}\right)=1$ defines the escape cone for internal radiation, $I_{\text {ext }}$ and $I_{\text {int }}$ are the external and internal luminescence intensities per unit area respectively, $\theta$ is the internal angle of incidence, and the factor of 2 accounts for the top and bottom surfaces of the solar-cell slab as illustrated in Fig. 3(a). If the slab is planar and has nonabsorbing surfaces, the excess spontaneous emission is not really lost. The internally emitted light will reflect back and forth indefinitely by total internal reflection between the top and bottom surfaces until it is finally reabsorbed by weak band-tail absorption. In principle the internal spontaneous emission is thus never really lost and the overall emission is diminished by the factor $n^{2}$. In reality, as a result of parasitic surface absorption, this scheme is not very practical.
A much more worthwhile arrangement is shown in Fig. 3(b) where all the internal spontaneous emission is coupled to the exterior by surface texturing. In this case all the internal emission is lost, but is compensated by a corresponding $2 n^{2}$ increase in the absorption path length for externally incident radiation which becomes internally trapped. ${ }^{10}$ This permits the semiconductor slab to be correspondingly thinner, while maintaining the same degree of absorption. But then the internal spontaneous emission is diminished by a simple reduction of semiconductor volume. Therefore the light-trapping geometry of Fig. 3(b) is already optimized for inhibited spontaneous emission, and has many other benefits as well such as reducing the needed volume of semiconductor material, increasing the output voltage and the conversion efficiency. A geometry of the type shown in Fig. 3(b) recently broke all records $(28.2 \%)$ for solar-cell efficiency. ${ }^{11}$ Therefore, I must conclude that inhibited spontaneous emission is already incorporated to the maximum permissible degree in the most modern solar-cell designs.

I conclude that inhibited spontaneous emission due to an electromagnetic band gap can be a significant effect in solid-state physics and may someday have a role to play in the study of semiconductor lasers.

I would like to thank J. M. Worlock and J. Salzman for valuable discussions.

${ }^{1}$ E. M. Purcell, Phys. Rev. 69, 681 (1946).

${ }^{2}$ R. G. Hulet, E. S. Hilfer, and D. Kleppner, Phys. Rev. Lett. 55, 2137 (1985).

${ }^{3}$ G. Gabrielse and H. Dehmelt, Phys. Rev. Lett. 55, 67 (1985)

${ }^{4}$ R. J. Nelson and R. G. Sobers, J. Appl. Phys. 49, 6103 (1978).

${ }^{5}$ H. Kogelnik and C. V. Shank, J. Appl. Phys. 43, 2328 (1972).

${ }^{6}$ E. Yablonovitch, R. Bhat, J. P. Harbison, and R. A. Logan, Appl. Phys. Lett. 50, 1197 (1987).

${ }^{7}$ H. A. Haus and C. V. Shank, IEEE J. Quantum Electron 12, 532 (1976); S. L. McCall and P. M. Platzman, IEEE J. Quantum Electron 21, 1899 (1985).

${ }^{8}$ E. Yablonovitch and E. O. Kane, J. Lightwave Technol. 4, 504, 961(E) (1986); A. R. Adams, Electron. Lett. 22, 249 (1986).

${ }^{9}$ A. Yariv and P. Yeh, Optical Waves in Crystals (Wiley, New York, 1984), p. 174.

${ }^{10}$ E. Yablonovitch, J. Opt. Soc. Am. 72, 899 (1982).

${ }^{11}$ R. A. Sinton, Y. Kwark, J. Y. Gan, and R. M. Swanson, IEEE Electron Device Lett. 7, 567 (1986). 